\title{
Advances in EELS Instrumentation: A New Design High-Vacuum Parallel EELS System
}

\author{
R.D. Twesten, ${ }^{*}$ C.G. Trevor, ${ }^{*}$ M.M.G. Barfels, ${ }^{*}$ Y. Sato, ${ }^{*}$ N.K. Menon, ${ }^{*}$ \\ P.J. Thomas, ${ }^{*}$ and A.J. Gubbens* \\ *Gatan Inc., 5794 W. Las Positas Blvd., Pleasanton, CA 94588
}

Electron energy-loss spectroscopy (EELS) has become a common feature in most TEM labs allowing the routine analysis of elemental composition, local bonding and optical properties at the sub-nanometer and some cases atomic scale. In this abstract we present the primary design features of a new high-vacuum dedicated EELS system that exploits recent advances in energy filtered imaging equipment [1] which have made available new acquisition modalities not previously available in a commercial system.

The primary design concern is the vacuum requirements. A focused STEM probe acts as a local deposition source for contamination on the sample fed by any source of volatile material in the vacuum or on the surface of the sample $[2,3]$. In most dedicated STEM system, the detector chamber shares a high conductance path with the specimen vacuum raising the vacuum requirements of the spectrometer system. For the present system, we have achieved a clean vacuum system by minimizing the number of o-rings, and providing differential pumping of the spectrometer. Most important feature, however, is the ability to vacuum bake the spectrometer to $140^{\circ} \mathrm{C}$ along with the rest of the STEM by employing a removable CCD detector system . The STEM vacuum is isolated from the CCD camera by a fiber optic plate which can faithfully transmit the electron scintillator signal to the CCD camera. The CCD camera is in a separate vacuum chamber to allow cooling of the chip to $-20{ }^{\circ} \mathrm{C}$, and this chamber can be removed while the rest of the system in being vacuum baked.

The electron-optical design of current generation Gatan EELS systems is based largely on the PEELS system developed by Krivanek and co-workers [4, 5], but substantial improvements in software, electronics and detectors have progressed over the years. The spectrometer presented here is based on the GIF Quantum ${ }^{\circledR}$ design [1] and makes a radical departure from the original PEELS design. A block diagram of the system is shown in figure 1. A key design improvement is the use of a straight faced gradient prism surrounded by 3 dodecapole lenses for aberration correction. These lenses are tuned to give a real, focused spectrum past the prism allowing the use of a knife edge beam trap limiting scattered electrons in the system and to serve as a clean beam stop for a high-speed electrostatic shutter higher in the spectrometer. Past the focused spectrum are 4 dodecapole lenses that project the final spectrum on the detector at varying dispersions with the lowest being $14 \mu \mathrm{m} / \mathrm{eV}$ (1.0 $\mathrm{eV} /$ pixel) allowing over $2000 \mathrm{eV}$ energy range to be projected on the detector. The axial ray for a dispersion of $140 \mu \mathrm{m} / \mathrm{eV}$ is shown in figure 2 The use of multipole lenses in the projector stack allows the dispersion to be varied without spectrum rotation or changing the width of the spectrum, which is fixed at $2.8 \mathrm{~mm}$, resulting in optimal readout of the CCD for all dispersions. The multipoles make possible the correction of distortions and non-linearity of the spectrum which is not possible for round lenses or quadrupole only based systems.

The spectrometer design allows the use of the fast electrostatic shutter, fast voltage offset and DualEELS systems developed for the GIF Quantum ${ }^{\circledR}$. The system also shares the high-speed stacked readout modes of the GIF Quantum $^{\circledR}$ giving $1 \mathrm{kHz}$ spectral readout for high-speed STEM-EELS mapping. 
1. A.J. Gubbens, et al., Ultramicroscopy 110, (2010), 962

2. L. Reimer and M. Wächter, Ultramicroscopy 3 (1978) 169

3. J. J. Hren, Introduction to Analytical Electron Microscopy, Plenum Press, (1979), Editors: Hren, Joy, Goldstein

4. O.L. Krivanek, Ultramicroscopy 28, (1989), 118

5. O.L. Krivanek, et al., Mircosc.Microanal. Microstruct. 2 (1991) 315

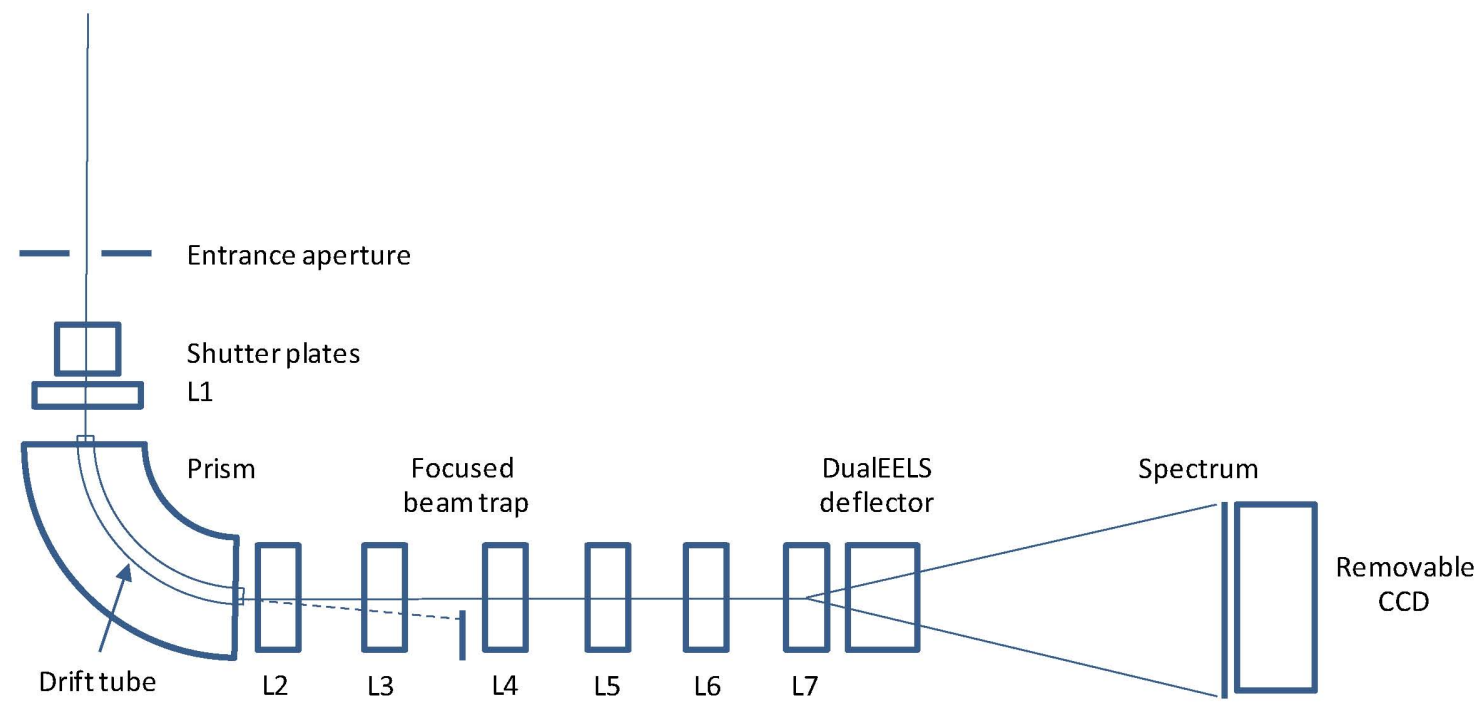

Figure 1. Schematic diagram of high-vacuum spectrometer. All lenses L1-7 are of the independently controlled multipole design giving all multipole functions up to dodecapole. The prism and lenses L1-3 create a focused spectrum inside the spectrometer allowing the use of a sharp beam trap to reduce scatter in the system. The fiber optically coupled CCD camera is in a separate vacuum and can be removed without breaking vacuum allowing the entire system to be baked to $140^{\circ} \mathrm{C}$.

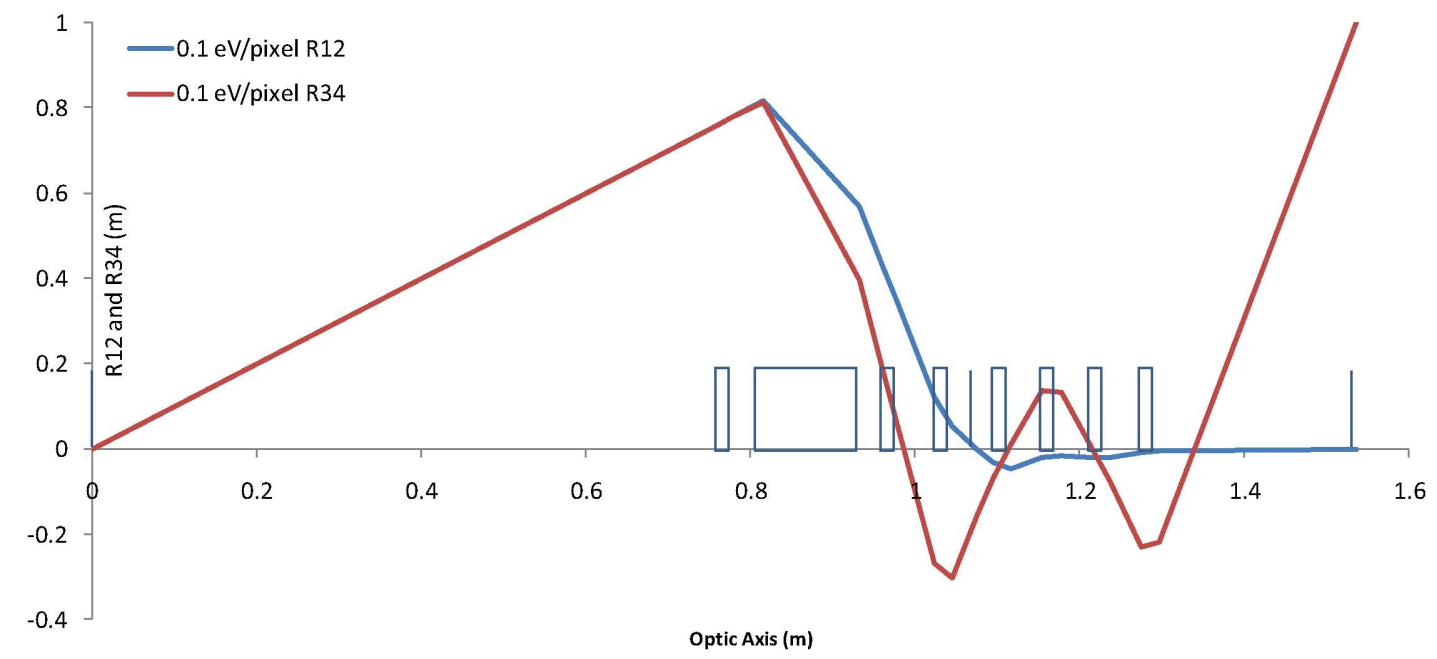

Figure 2. Axial rays of the spectrometer at $0.14 \mathrm{~mm} / \mathrm{eV}(0.1 \mathrm{eV} /$ pixel $)$ dispersion for $200 \mathrm{kV}$ primary beam. Rays are shown in standard transport notation. The non-dispersive ray is defocused to a $2.8 \mathrm{~mm}$ width to improve dynamic range on the CCD detector. The boxes show the position of the optical elements from figure 1 . 